\title{
The Subventricular Zone in the Immature Piglet Brain: Anatomy and Exodus of Neuroblasts into White Matter after Traumatic Brain Injury
}

\author{
Beth A. Costine $^{a, c}$ Symeon Missios ${ }^{d}$ Sabrina R. Taylore Declan McGuone ${ }^{b}$ \\ Colin M. Smith ${ }^{a}$ Carter P. Dodge ${ }^{f}$ Brent T. Harris ${ }^{g, h}$ Ann-Christine Duhaime ${ }^{a, c}$ \\ Departments of a Neurosurgery and ${ }^{b}$ Pathology, Massachusetts General Hospital, and ${ }^{c}$ Department of \\ Neurosurgery, Harvard Medical School, Boston, Mass., 'Department of Neurosurgery, Cleveland Clinic, Cleveland, \\ Ohio, e Department of Physical Medicine and Rehabilitation, Spaulding Rehabilitation Hospital, Charlestown, Mass., \\ ${ }^{f}$ Department of Anesthesiology, Children's Hospital at Dartmouth, Dartmouth Medical School, Lebanon, N.H., and \\ Departments of 9 Neurology and h Pathology, Georgetown Medical School, Washington, D.C., USA
}

\section{Key Words}

Traumatic brain injury - Subventricular zone .

Neurogenesis · Neuroblasts

\begin{abstract}
Stimulation of postnatal neurogenesis in the subventricular zone (SVZ) and robust migration of neuroblasts to the lesion site in response to traumatic brain injury (TBI) is well established in rodent species; however, it is not yet known whether postnatal neurogenesis plays a role in repair after TBI in gyrencephalic species. Here we describe the anatomy of the SVZ in the piglet for the first time and initiate an investigation into the effect of TBI on the SVZ architecture and the number of neuroblasts in the white matter. Among all ages of immaturity examined the SVZ contained a dense mesh network of neurogenic precursor cells (doublecortin+) positioned directly adjacent to the ependymal cells (ventricular SVZ, Vsvz) and neuroblasts organized into chains that were distinct from the Vsvz (abventricular SVZ, Asvz). Though the architecture of the SVZ was similar among ages, the areas of Vsvz and Asvz neuroblast chains declined with age. At postnatal day (PND) 14 the white matter tracts have a tremendous number of individual neuroblasts. In our scaled cortical
\end{abstract}

impact model, lesion size increased with age. Similarly, the response of the SVZ to injury was also age dependent. The younger age groups that sustained the proportionately smallest lesions had the largest SVZ areas, which further increased in response to injury. In piglets that were injured at 4 months of age and had the largest lesions, the SVZ did not increase in response to injury. Similar to humans, swine have abundant gyri and gyral white matter, providing a unique platform to study neuroblasts potentially migrating from the SVZ to the lesioned cortex along these white matter tracts. In piglets injured at PND 7, TBI did not increase the total number of neuroblasts in the white matter compared to uninjured piglets, but redistribution occurred with a greater number of neuroblasts in the white matter of the hemisphere ipsilateral to the injury compared to the contralateral hemisphere. At 7 days after injury, less than $1 \%$ of neuroblasts in the white matter were born in the 2 days following injury. These data show that the SVZ in the piglet shares many anatomical similarities with the SVZ in the human infant, and that TBI had only modest effects on the SVZ and the number of neuroblasts in the white matter. Piglets at an equivalent developmental stage to human infants were equipped with the largest SVZ and a tremendous number of neuroblasts in the white matter, which may be sufficient in

\section{KARGER 125}

(c) 2015 S. Karger AG, Base

0378-5866/15/0372-0115\$39.50/0

E-Mail karger@karger.com

www.karger.com/dne
Dr. Beth A. Costine, $\mathrm{PhD}$

Massachusetts General Hospital, Harvard Medical School

Building 149, 13th Street, Room 3.405

Charlestown, MA 02114 (USA)

E-Mail bcostine@mgh.harvard.edu 
lesion repair without the dramatic stimulation of neurogenic machinery. It has yet to be determined whether neurogenesis and migrating neuroblasts play a role in repair after TBI and/or whether an alteration of normal migration during active postnatal population of brain regions is beneficial in species with gyrencephalic brains.

(c) 2015 S. Karger AG, Basel

\section{Introduction}

The main sites of postnatal neurogenesis include the subventricular zone (SVZ) surrounding the lateral ventricles and the subgranular zone of the dentate gyrus of the hippocampus [1]. The SVZ is a mitotically active region persisting beyond fetal development serving as a source of new glia, neurons and oligodendrocytes. In species in which olfaction is an important sense such as rodents [2], dogs [3] and rabbits [4], large numbers of newly generated neurons migrate through the SVZ into the rostral migratory stream (RMS), a route leading to the olfactory bulbs where they replenish GABAergic interneurons throughout life. A similar migration to the olfactory bulbs occurs in humans but on a smaller scale that declines precipitously with age [5]. In the postnatal and adult pig (Sus scrofa), proliferating cells have been demonstrated in the SVZ and dentate gyrus, and neuronal stem cells from the SVZ produce both neurons and glia in vitro [6-8]. However, the anatomical structure of the SVZ in the piglet has not yet been described.

In rodent models, a role for postnatal neurogenesis has been described after heterogeneous neural insults, including traumatic brain injury (TBI), seizures, stroke, ischemia, and withdrawal from alcohol dependence [914], indicating that neurogenesis and attendant migration is a common strategy applied in response to diverse central nervous system insults within these species. Immature neurons recruited to the area of injury might replace damaged neurons by synaptically integrating and/ or might acutely support surviving neurons via the secretion of neurotrophins $[9,15,16]$. However, in infants and children it remains to be determined whether TBI stimulates neurogenesis and the migration of immature neurons, whether these cells successfully arrive at the lesional brain tissue and whether they participate in lesion repair [17]. It is not known whether neurogenesis is stimulated and/or migration is directed to the lesion in a gyrencephalic model of TBI. Further, if neurogenesis or the migration of neuroblasts is stimulated by TBI in hu- mans at various stages of development, it is not known whether the recruitment of neuroblasts to the injury is beneficial. Posttraumatic alterations of neuroblast migration may contribute to the development of posttraumatic epilepsy or redirect neuroblasts requisite for normal postnatal brain development.

Because of the limiting nature of studies performed on human tissue after TBI, large animal models might work in concert with human studies and help to determine whether postnatal neurogenesis aids in repair after injury or, alternatively, provides a substrate that potentiates the long-term neurological sequelae complicating head injury [17-19]. Indeed, over 100 therapies have been identified that ameliorate brain trauma in rodents, yet none have effectively translated into the management of brain trauma in children [20], thus highlighting the urgent need to study this potential inherent brain repair mechanism in a model in which anatomy and the pattern of neurodevelopment better parallel those of children. In contrast to the lissencephalic brain of the rodent, which contains a limited volume of white matter, the brain of the domestic pig is gyrencephalic, similar to humans [18]. The human brain shares more developmental similarities with the piglet brain than it does with the rodent or primate brain in terms of its maximal phase of growth, including cellular proliferation and myelination peaking around the time of birth $[18,21,22]$.

We have previously demonstrated that a cortical impact delivering the same biomechanical strain scaled among ages results in larger contusions in older subjects $[23,24]$. For reasons yet unknown, piglets injured at a developmental stage comparable to human infants (postnatal day, PND 7) develop little-to-no contusional injury, whereas older piglets that are developmentally comparable to human preadolescents (4 months of age) develop large lesions [23]. It is unknown whether the variation in lesion development among ages might be due to differences in age-dependent responses and cell death pathways or whether the repair mechanisms differ, including the basal rate of neurogenesis, increased inherent reparative capacity in younger subjects or an ability to bolster neurogenesis in response to TBI. Here, our aims were as follows: (1) to describe the SVZ of the piglet, (2) to determine the effect of cortical impact on the SVZ at three stages of development (infant: PND 7; toddler: 1 month; preadolescent: 4 months) when examined 1 week after injury and (3) to determine whether cortical impact increases the number of putative migrating neuroblasts in the cerebral white matter in piglets injured at PND 7. 


\section{Experimental Procedures}

\section{General}

Yorkshire swine (Earle Parsons \& Sons, Inc., Hadley, Mass., USA) were housed in a temperature-controlled facility (21$22^{\circ} \mathrm{C}$ ) with a 12 -hour light cycle with lights on at 07:00. All protocols and procedures were in accordance with the guidelines of the American Veterinary Association and the National Institute of Health and were approved by the Institutional Animal Care and Use Committee at Dartmouth College or the Subcommittee of Research Animal Care at Massachusetts General Hospital.

Male and female swine underwent scaled cortical impact at PND 7, 1 month or 4 months of age under isoflurane anesthesia mixed with room air, as previously described [23, 24]. A craniectomy was performed, the dura was opened, and cortical impact was achieved via a spring-loaded device secured to the skull with an indentor tip scaled to the size of the brain in three dimensions for each age of animal such that $1 \%$ of the volume of the brain was rapidly displaced over $4 \mathrm{~ms}$, keeping mechanical input (strain) identical among ages [23]. The rostral gyrus on the right hemisphere, the somatotopic area of the somatosensory cortex corresponding to the snout, was injured, resulting in a clinically silent but pathologically significant lesion that has previously been found to increase with increasing age [23]. On day 7 after injury the piglets were deeply anesthetized and transcardially perfused with saline $(0.9 \%)$ and then phosphate-buffered formalin (PBF; $10 \%)$, and brains were collected.

The Department of Pathology at Georgetown University Hospital supplied the tissue for the single example of the SVZ in human infants. Brain tissue was collected in accordance with the guidelines of the institutional review board of Georgetown University Hospital from a 5-month-old male infant who died due to reasons other than TBI (cystic fibrosis) and had no history of TBI. The block of tissue (approximately $3 \times 7.5 \mathrm{~cm}^{2}$ ) was dissected and fixed in $10 \% \mathrm{PBF}$, embedded in paraffin, and $8-\mu \mathrm{M}$ sections were probed for doublecortin (DCX), as described below.

\section{Analysis of the SVZ among Ages after TBI}

As previously described, $10-\mu \mathrm{M}$ sections were taken every 0.25 $\mathrm{mm}$ and stained with hematoxylin and eosin [23]. For each subject $(n=67)$, two sections were chosen corresponding to $25 \%$ (anterior; A 8.5-12.5 mm) and 50\% (middle; A 5.0-8.5 mm) of the span of the lesion, with $50 \%$ at the center of the lesion. Photomicrographs of the images were obtained with an Olympus BX50 light microscope (Center Valley, Pa., USA) and an SPOT Insight 2MP Firewire Color Mosaic camera (Diagnostic Instruments, Sterling Heights, Mich., USA). The morphometry of the SVZ was determined using Analytical Imaging Station (Imaging Research Inc., St. Catherines, Ont., Canada). The area of the ventricular SVZ (Vsvz) and total area of neuroblast chains in the abventricular SVZ (Asvz; high density of cells that take up hematoxylin that correspond to DCX+ [25]) was determined with the 'outline function' by observers blinded to the subject age and hemisphere. For each parameter only those subjects in whom both hemispheres could be analyzed were included in the data set. Sections in which the lesion resulting from the cortical impact extended into the SVZ were excluded, resulting in a total of 40 subjects.
Analysis of the Architecture of the SVZ and Quantification of Migrating Immature Neurons

PND-7 male piglets $(\mathrm{n}=14)$ were randomly assigned to undergo scaled cortical impact $(n=8)$ or sham surgery/naïve treatment ( $\mathrm{n}=3$ sham; $\mathrm{n}=3$ naïve) via coin flip. In this first attempt at detecting migrating neuroblasts in piglets after TBI, the sample size and the time required between injury and examination of neuroblasts in the white matter was based on previous rodent studies $[2,13]$, since the time required for potentially migrating neuroblasts generated in the SVZ to reach the gyral white matter is not known in piglets. Piglets received a cortical impact, as described above, or sham surgery where the indentor was not deployed. Immediately after cortical impact and once a day in the 2 days following surgery they were given an intraperitoneal injection of $50 \mathrm{mg} /$ $\mathrm{kg}$ of 5-bromo-2-deoxyuridine (BrdU; $50 \mathrm{mg} / \mathrm{kg}$ ), an analog of thymidine, to label cells undergoing cell division, for a total of 3 injections $[13,26]$. Naïve animals were injected with BrdU at the same time. A single piglet of the same age received neither surgery nor BrdU for analysis of the migratory streams in the sagittal plane. The piglets were euthanized and brains were collected 7 days after injury on PND 14, as described above - 1 injured piglet was euthanized prior to the end of the experiment due to pneumonia and diarrhea unrelated to the treatments, and 1 sham piglet was excluded upon discovery of a hematoma at the site of the sham surgery.

After postfixation three adjacent 5-mm coronal slabs [27] were collected, starting at the anterior edge of the rostral gyrus and continuing caudally, encompassing the region of the injury and the underlying SVZ. In the piglet used for analysis of the RMS, the brain was sliced in the sagittal plane of the 5-mm block containing the olfactory bulb and was interrogated for the RMS (lateral level, L $3.8-6.80 \mathrm{~mm}$ ). Hemispheres were separated and postfixed in $\mathrm{PBF}$ overnight at $4^{\circ} \mathrm{C}$. The slabs were cryoprotected with PBS with $30 \%$ sucrose (2-3 days), embedded in optimum cutting temperature compound (Tissue-Tek ${ }^{\circledR}$ ), frozen, and stored at $-80^{\circ} \mathrm{C}$. Frozen sections $(50 \mu \mathrm{M})$ of the 5 -mm slabs encompassing the injury on the rostral gyrus and the underlying SVZ (anterior plane, A $1.5-14.5 \mathrm{~mm}$ ) and the corresponding regions in the uninjured hemispheres were systematically made on a cryostat (Leica Microsystems, Deerfield, Ill., USA). Due to the large size of the piglet brain, every other section was mounted on poly-L-lysine-coated microscope slides (Superfrost ${ }^{\circledR} /$ Plus, Fisherbrand ${ }^{\circledR}$; Fisher Scientific, Pittsburgh, Pa., USA), resulting in approximately $100 \mathrm{sec}-$ tions per hemisphere. The sections were dried and stored at $-80^{\circ} \mathrm{C}$.

Immunofluorescence was used to assess the anatomy of the SVZ and neuroblasts with a migratory phenotype in the white matter with a previous protocol [28] adapted to frozen sections and antigen retrieval for BrdU. Between all steps, the sections were washed 3 times in $0.1 \mathrm{M}$ tris-buffered saline (TBS; $1 \mathrm{M}$ Tris$\mathrm{HCl}, 1.5 \mathrm{M} \mathrm{NaCl} ; \mathrm{pH}$ 7.5). The mounted tissue was rehydrated in TBS followed by permeabilization in 1\% Triton X-100 (ICN Biomedicals, Aurora, Ohio, USA) in TBS (TBST) for $20 \mathrm{~min}$. Incubation in $50 \%$ formamide $/ 2 \mathrm{x}$ saline sodium citrate $(0.3 \mathrm{M} \mathrm{NaCl}$, $30 \mathrm{mM}$ sodium citrate) for $2 \mathrm{~h}$ at $65^{\circ} \mathrm{C}$ followed by incubation in $2 \mathrm{~N} \mathrm{HCl}$ at $37^{\circ} \mathrm{C}$ for $30 \mathrm{~min}$, and equilibration in $0.1 \mathrm{M}$ boric acid was performed for $10 \mathrm{~min}$ for antigen retrieval for BrdU. For tissue not probed for BrdU, antigen retrieval was accomplished by heating sections in $10 \mathrm{mM}$ citric acid buffer ( $\mathrm{pH}$ 6.0) in a pressure cooker (DAKO; Carpinteria, Calif., USA) followed by equilibra- 
tion to room temperature for $30 \mathrm{~min}$. The sections were blocked in 3\% donkey serum/TBST (TBSTD; Invitrogen, Carlsbad, Calif., USA) for $30 \mathrm{~min}$ before incubation with antibodies for DCX (goat polyclonal anti-human DCX; Santa Cruz Biotechnology, Santa Cruz, Calif., USA; sc-8066; 1:100), BrdU (rat monoclonal anti-BrdU; Abcam, Cambridge, Mass., USA; ab6326; 1:75), GFAP (mouse monoclonal anti-human; DakoCytomation, Glostrup, Denmark; MO761; 1:100), Ki-67 (mouse monoclonal anti human Ki-67; BD Pharmigen, San Jose, Calif., USA; 556003; $1: 100$ ) or NeuN (mouse monoclonal anti-mouse NeuN; Millipore, Billerica, Mass., USA; MAB377; 1:100) overnight at room temperature. The sections were washed and incubated with secondary antibodies (Alexa Fluor ${ }^{\circledR} 488$ or 568 donkey anti-goat, anti-rat or anti-mouse IgG; Invitrogen; 1:200) diluted in TBSTD for $2 \mathrm{~h}$ at room temperature, incubated in 4',6-diamidino-2-phenylindole (DAPI, $\leq 2 \mathrm{~min}$; Invitrogen Molecular Probes, Eugene, Oreg., USA) or TO-PRO ${ }^{\circledR}-3$ iodide (TOPRO; $1 \mu \mathrm{M} ; 10$ min; Life Technologies, Grand Island, N.Y., USA) to distinguish nuclei, washed, and cover-slipped with Vectashield or Hard Set Mounting Medium (Vector Laboratories, Inc., Burlingame, Calif., USA). Negative control sections excluded primary antibodies. This antibody to DCX has been previously validated in our laboratory and does not colabel with reactive microglia (HLA-DP; mouse monoclonal anti-human HLA-DP; DakoCytomation; M0775; 1:100), macrophages (CD68; mouse monoclonal antihuman CD68; DakoCytomation; M0876; 1:100) or astrocytes (GFAP) [17].

For anatomical studies, the sections were visualized with a Nikon Eclipse TE2000S Epifluorescent microscope (Nikon, Melville, N.Y., USA) and images were obtained with an Optronics Digital MicroFire microscope-camera, or the sections were visualized with a Zeiss Axiovert 200 and images were obtained using a Zeiss LSM 510 META Scanning Confocal Microscope System. Colabeling of BrdU and DCX within the same cell was confirmed by analyzing $\mathrm{z}$-stacks at $40 \times$ or $63 \times$ using a Zeiss LSM 510 META Scanning Confocal Microscope System.

\section{Quantification of Migrating Immature Neurons in the White \\ Matter}

For quantification of DCX+ cells migrating through all major hemispheric white matter tracts, sections every $1,200 \mu \mathrm{M}$ apart after a random start within the first 10 sections until the end of the SVZ were used for the detection of DCX+ cells of each hemisphere, resulting in 6-8 sections analyzed per hemisphere/piglet. In the white matter, DCX+ cells were counted systematically using a motorized stage and a Zeiss Axiovert 200 by observers blinded to the treatment. DCX+ cells were characterized by an elongated morphology and often had multiple neurites. A cell was counted as a single cell if neurites were continuous and separately if neurites were discontinuous. The white matter in each hemisphere was considered a single disector and the number of DCX+ cells was quantified using the disector pair approach of stereology [29]. The total number of DCX+cells among the 6-8 sections (random start, $1,200 \mu \mathrm{M}$ apart through the SVZ) in the white matter of each hemisphere was estimated using the formula $\mathrm{N}_{\text {objects }}=\sum \mathrm{Q}^{-}{ }_{\mathrm{obj}}\left(\mathrm{F}_{1}\right)\left(\mathrm{F}_{2}\right)$, where $F_{1}=1 /$ section sampling fraction (where section sample fraction $=$ reference space thickness/total thickness of disector pairs) and F2 $=1$ /thickness sampling fraction (where thickness sampling fraction $=$ disector height/section thickness) [29]. The coefficient of variation was 0.32 , the coefficient of error was 0.12 and biologi- cal variation was 0.297 , resulting in $92.6 \%$ of the variation due to biological variation and $7.4 \%$ of the variation due to variation among sections, indicating that our number of sections sampled was sufficient [29].

\section{Statistics}

The effects of age and hemisphere in relation to the injury and the interaction on the area of the Vsvz and total neuroblast chain area in the Asvz were tested with a two-way analysis of variance with the general linear model of SAS (Enterprise Guide 4.3 On Demand; Cary, N.C., USA) followed by paired t tests between ipsilateral and contralateral hemispheres within each age. Differences in lesion size among ages in a subset of piglets previously published [23] in which we analyzed the SVZ here were tested by unpaired $t$ tests. The total estimated numbers of migrating neurons in naïve and sham animals were not different and were therefore combined. To determine the effect of injury on injured versus control piglets unpaired Student's t tests were performed to compare the difference in the number of DCX+ cells in the white matter of the ipsilateral and contralateral hemispheres, as well as the total number of migrating neurons per piglet (ipsilateral + contralateral). Differences in the number of neuroblasts in the white matter between hemispheres (ipsilateral vs. contralateral) within treatment (injured or control) were determined via paired Student's t tests. Graphs depict means \pm SEM. $p$ values $<0.05$ were considered significant; $p$ values between 0.05 and 0.07 were noted as a tendency to be significant.

\section{Results}

\section{SVZ Anatomy in Uninjured PND-14 Piglets}

The SVZ was examined in the anterior lateral ventricle of uninjured PND-14 piglets (fig. 1, 2). Along the lateral ventricle a thin Vsvz is observed adjacent to the ventricle. Adjacent to the anterior portion of the lateral ventricle (fig. 1a, b) the Vsvz expands into an Asvz. The Asvz is continuous with the Vsvz, with a dense network of glial cells (GFAP+) and neuroblasts (DCX+) with an individual migratory phenotype. Similar to the human infant, two distinct populations of neuroblasts exist in the piglet - Vsvz adjacent to the ventricle and Asvz apart from the ventricle (fig. 1i).

The SVZ of the PND-14 piglet is a heterogeneous structure containing proliferating cells. The anterior SVZ is bordered by the head of the caudate nucleus inferiorly, the genu and body of the corpus callosum dorsally, the centrum semiovale dorsalaterally, and the anterior limb of the internal capsule laterally (fig. 1a-h). Within the SVZ, the following four distinct regions are identified: (1) a single layer of ependymal cells lining the lateral ventricle, (2) a dense, thick layer of DCX+ and GFAP+ cells directly adjacent to the ependyma - the Vsvz, (3) the parenchyma of the SVZ containing individual DCX+ and GFAP+ cells and (4) most laterally, a region containing 


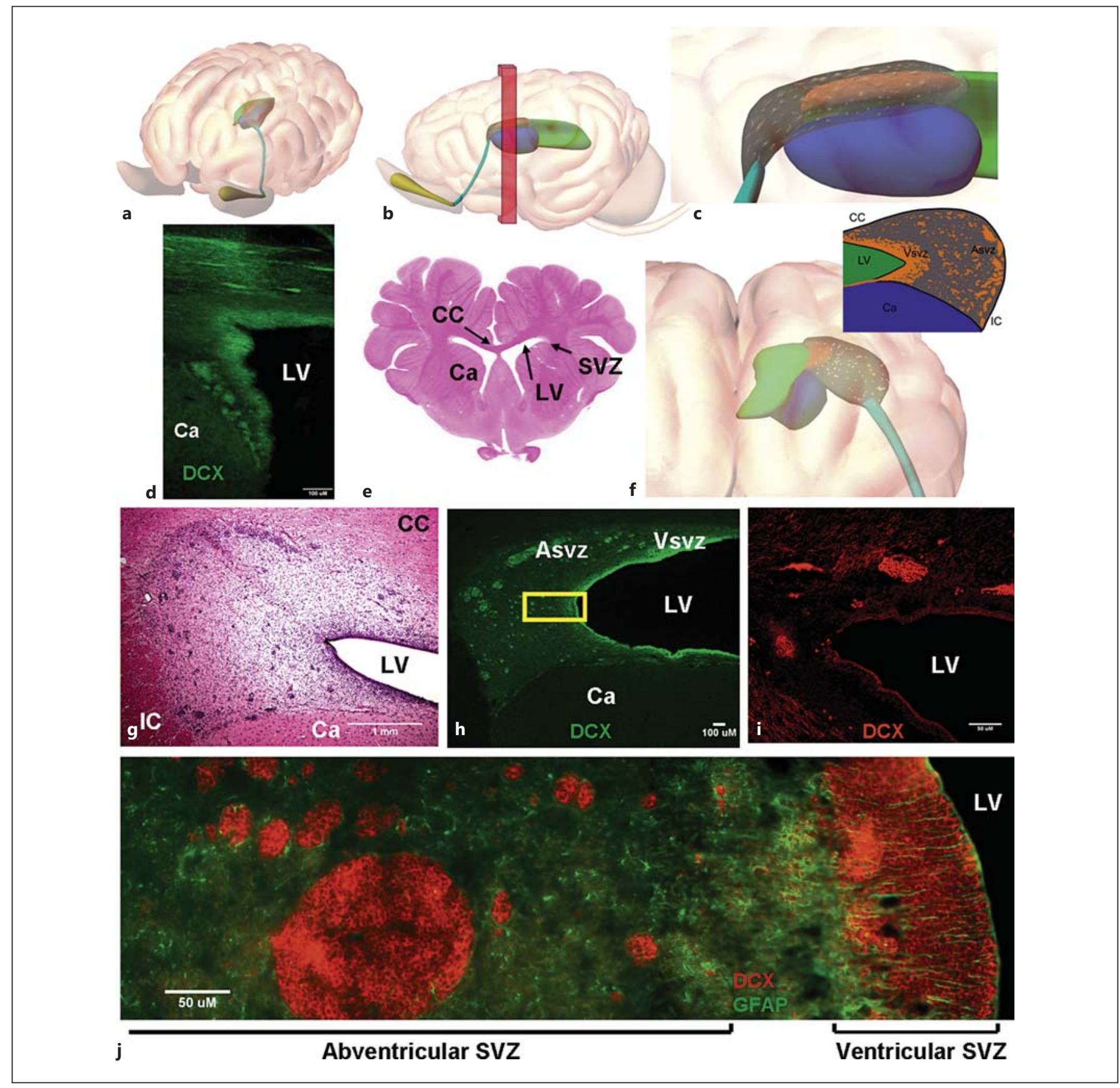

Fig. 1. Architecture of the uninjured SVZ of the PND-14 piglet and comparison to the human infant. $\mathrm{LV}=$ Lateral ventricle; $\mathrm{CC}=$ corpus callosum; $\mathrm{Ca}=$ caudate; $\mathrm{IC}=$ internal capsule. $\mathbf{a}, \mathbf{b} 3 \mathrm{D}$ illustrations of the SVZ along the anterior lateral ventricle (green) in the piglet, indicating the location of coronal sections in $\mathbf{e}, \mathbf{g}, \mathbf{h}$ (green: lateral ventricle; blue: caudate; orange: Vsvz; gray: Asvz; teal: RMS; yellow: olfactory ventricle). c Illustration of a sagittal view of the anterior SVZ corresponding to the sagittal section in d. The piglet $S V Z$ is a distinct anatomical feature containing glia $(\mathrm{GFAP}+)$ and neuroblasts (DCX+: marker of immature migrating neuroblasts; $\mathbf{d}, \mathbf{h}, \mathbf{j})$ that correspond to the hematoxylin staining (g). f $3 \mathrm{D}$ illustration of the coronal view of the SVZ and the $2 \mathrm{D}$ inset illustrate that the SVZ around the anterior lateral ventricle (green) is surrounded by the corpus callosum dorsally, the head of caudate (blue) ventrally, and the internal capsule laterally. The SVZ in the piglet has two portions - the Vsvz and the Asvz. $\mathbf{i}$ The SVZ in a 5-month-old human infant where the Vsvz and Asvz neuroblast chains are present and similar to the PND-14 piglet. $\mathbf{j}$ Higher magnification image of yellow box in $\mathbf{h}$. From the lateral ventricle out, directly under the ependymal layer, is a dense network of neuroblasts (Vsvz), a region dense with glia and, laterally, a region with chains of neuroblasts distant from the ventricle (Asvz, region of yellow box of $\mathbf{h}$ ). Scale bars: $\mathbf{g}=1 \mathrm{~mm} ; \mathbf{d}, \mathbf{h}=$ $100 \mu \mathrm{M} ; \mathbf{i}, \mathbf{j}=50 \mu \mathrm{M}$. 

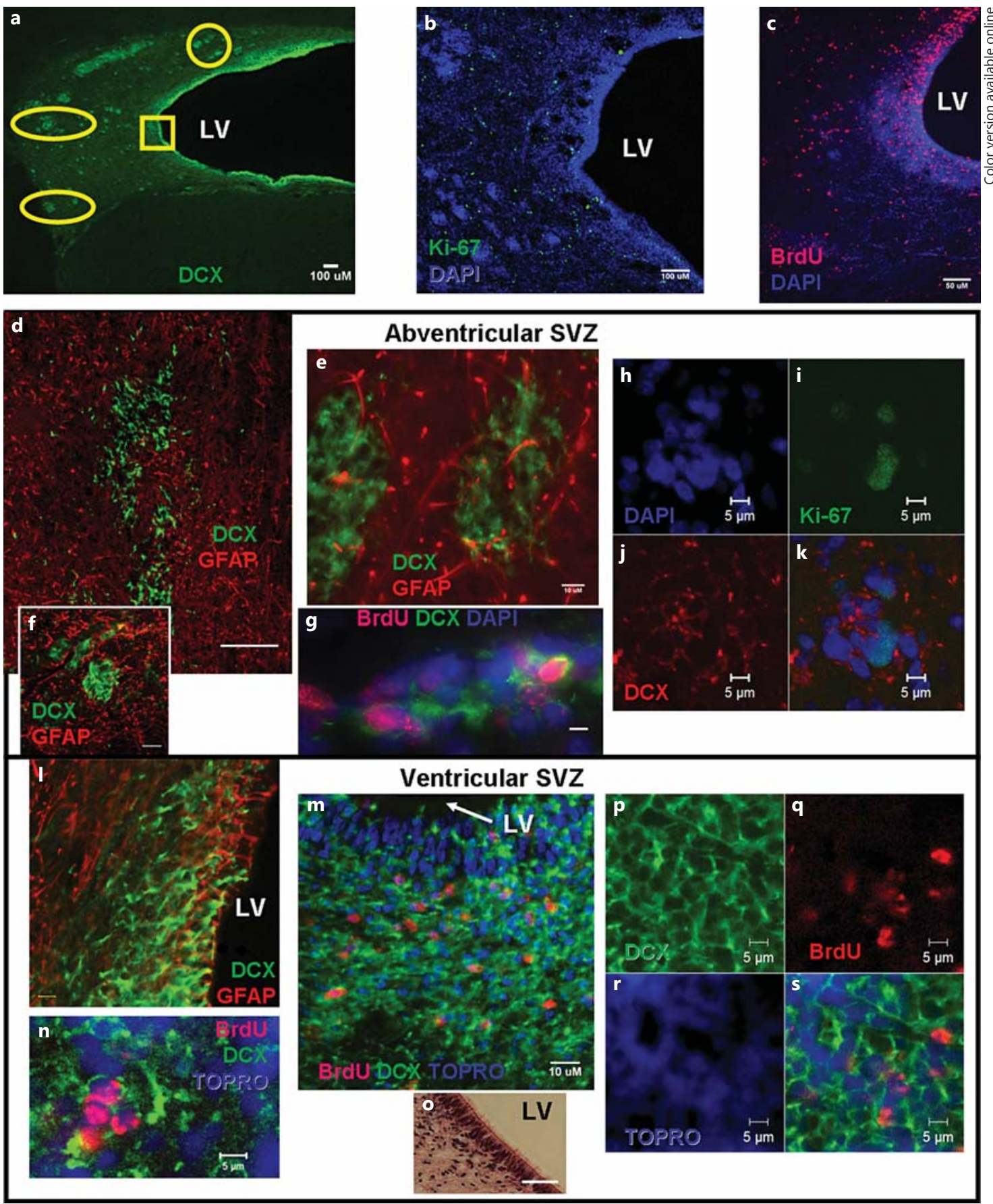

Fig. 2. Characteristics of the Vsvz and Asvz in the uninjured PND14 piglet. Coronal section with square indicating Vsvz, circle indicating dorsal Asvz, ovals indicating ventral Asvz (a). An abundance of proliferating cells are present throughout the SVZ of the PND-14 piglet as marked by Ki-67 (b) or the detection of BrdU administered at PND 7-9 (c). The chains of neuroblasts in the Asvz (upper panel) have varied characteristics according to location. d Neuroblast chains located in the dorsal Asvz (yellow circle, a; color in online version only) are loose in structure and have less contact with GFAP+ cells. e-h Abventricular neuroblast chains lo- cated ventrally (yellow ovals, a) are clustered tightly, have GFAP+ cells with a few processes wrapped around the chains (e) and are positive for markers of proliferation (BrdU, g; Ki-67, i, k). In the Vsvz (lower panel, yellow square in a), neuroblasts create a dense mesh network directly underlying the ependyma (pseudostratified, $\mathrm{H} \& \mathrm{E}, \mathbf{o}$ ) with GFAP+ processes extending between ependymal cells (I). Neuroblasts in the Vsvz are proliferative, as detected via BrdU administered on PND 7-8 (n, m, q, s). Scale bars: a, b, d, $\mathbf{f}=100 \mu \mathrm{M} ; \mathbf{c}=50 \mu \mathrm{M} ; \mathbf{e}, \mathbf{I}, \mathbf{m}=10 \mu \mathrm{M} ; \mathbf{o}=20 \mu \mathrm{M} ; \mathbf{g}-\mathbf{k}, \mathbf{n}, \mathbf{p}-\mathbf{s}=$ $5 \mu \mathrm{M}$. 
large chains of DCX+ cells in the Asvz (fig. 1g, h, j). The SVZ is mostly devoid of mature neurons, exhibiting only sporadic, small NeuN+ cells (data not shown). An abundance of proliferating cells are present throughout the SVZ of the PND-14 piglet, as detected by BrdU (injected at PND 7-9) and Ki-67 (fig. 2b, c).

The ependymal cells form a single layer of pseudostratified columnar, ciliated epithelium (fig. 20). Long cell processes extend from GFAP+ cells of the Vsvz and interdigitate with the ependymal cells (fig. 1j, 2l). A hypocellular gap layer devoid of DCX+cells between the ependymal cells and Vsvz, as occurs in human infants, was not observed. The DCX+ cells of the Vsvz tightly abut the ependyma as a dense, continuous mesh of DCX+ and GFAP+ cells (fig. 1j, 2l-n, p-s). Neuroblasts in the Vsvz are proliferative and positive for both BrdU (fig. $2 \mathrm{~m}, \mathrm{n}$, p-s) and Ki-67 (fig. 2b). Adjacent to the Vsvz is an area where neuroblast chains are absent, but individual DCX+ and GFAP+ cells are present (fig. 1j).

A striking characteristic of the piglet SVZ is the large number and size of chains of DCX+ cells located in the Asvz. Neuroblast chains in the ventral Asvz are round in both coronal and sagittal sections (fig. 1d, h), but in the dorsal Asvz most neuroblast chains are elongated in a migratory phenotype oriented anterior to posterior in the sagittal plane (fig. 1c, d). In coronal sections chains are spherical to oblong, typically occurring in a 3-10 (short axis) $\times 3-20$ neuroblast (long axis) array (fig. 2e). The organization of the chains differs according to their location within the Asvz (fig. 2d-f). Chains located ventrally (fig. 2a, yellow ovals) are compact and organized, containing spheroid DCX+ cells in both the sagittal and coronal plane. Each cluster has several GFAP+ cells with long processes embracing the chains (fig. 2e, f). Chains located in the dorsal Asvz (fig. 2a, yellow circle) are less organized and less compact; DCX+ cells are more elongated and have fewer associations with GFAP+ cells (fig. 2d). Piglets do not appear to have glial tubes where neuroblasts are completely ensheathed in glia, as occurs in rodents. Frequently, DCX+ cells with a migratory phenotype are observed adjacent to the loose chains, appearing to be moving out of the cluster (fig. 2d). Nuclei of neuroblasts (DCX+) in the chains of the Asvz were positive for BrdU (fig. 2g) and Ki-67 (fig. 2h-k).

Radial glia or radial-like glia were observed in the SVZ of some PND-14 piglets via immunodetection of GFAP. They initially radiated perpendicular from the lateral ventricle and then projected laterally through the SVZ but were not observed to project through the adjacent white or gray matter (fig. 3a, b, d). A portion of DCX+ cells appear to align with the radial glia (fig. $3 c$, d).

Neurogenesis after TBI in Piglets

The borders of the SVZ and the distinction between the adjacent brain regions varied among regions. The ventral border of the SVZ was distinct around the head of the caudate nucleus (fig. $3 \mathrm{f}, \mathrm{g}$ ) but indistinct dorsally (blending into the white matter of the corpus callosum) and dorsolaterally (blending with the centrum semiovale), with an abundant quantity of neuroblasts in a migrating phenotype in both the SVZ and the adjacent white matter (fig. 3e, f, h).

\section{Migratory Streams in Uninjured PND-14 Piglets}

Piglets have prominent olfactory bulbs rostral to the cortex, measuring approximately $1.5 \times 2 \mathrm{~cm}$ (width $\times$ height), comprising a significant portion of the size of the brain, which measures $5.5 \times 6.5 \mathrm{~cm}$ (width $\times$ length; piglets injured at 1 month old; fig. 4a). In sagittal sections the long chains of neuroblasts around the ventricle dorsally in the SVZ are continuous, with a stream of long chains of neuroblasts over the caudate rostral to the ventricle (fig. 4b, c). By mid-caudate the RMS has numerous immense chains of DCX+ cells in a 20-30 neuroblast (short axis) $\times 40-50$ neuroblast (long axis) array. Once past the caudate, neuroblasts descend between the putamen and claustrum in the white matter of the external capsule at L $5.6 \mathrm{~mm}$ [30] (fig. 4c, e). Ventral to the putamen, the RMS continues to deflect downward and moves laterally to $\mathrm{L} 4.0 \mathrm{~mm}$ [30] in the white matter tract, projecting to the olfactory ventricle, which is located closer to the anterior tip of the lateral ventricle laterally and thus travels a shorter distance than if the RMS projected from the SVZ to the olfactory ventricle in the same sagittal plane (fig. $4 \mathrm{~b}, \mathrm{c}, \mathrm{i}$ ).

The characteristics of the putative migrating neuroblasts and neuroblast chains differ according to region in the RMS. Near the lateral ventricle the chains are oriented anterior to posterior in the sagittal plane. These chains of migrating neuroblasts increase in size as they move rostrally away from the lateral ventricle and SVZ and into the RMS (fig. 4c, d) but stay in intermittent chains and are not continuous. Once into the external capsule, adjacent to the putamen, the chains appear to diminish in size and align with the gray matter in between the striatopallidal fibers (pencils of Wilson) and the claustrum (fig. 4c, f). After descending past the putamen the intermittent neuroblast chains continue moving laterally in small clusters along a white matter tract leading to the olfactory ventricle (fig. 4c, g).

In the sagittal plane putative migrating neuroblasts and neuroblast chains are primarily restricted to specific pathways, with the main path being the RMS, but additional routes were observed. An additional migratory stream may 


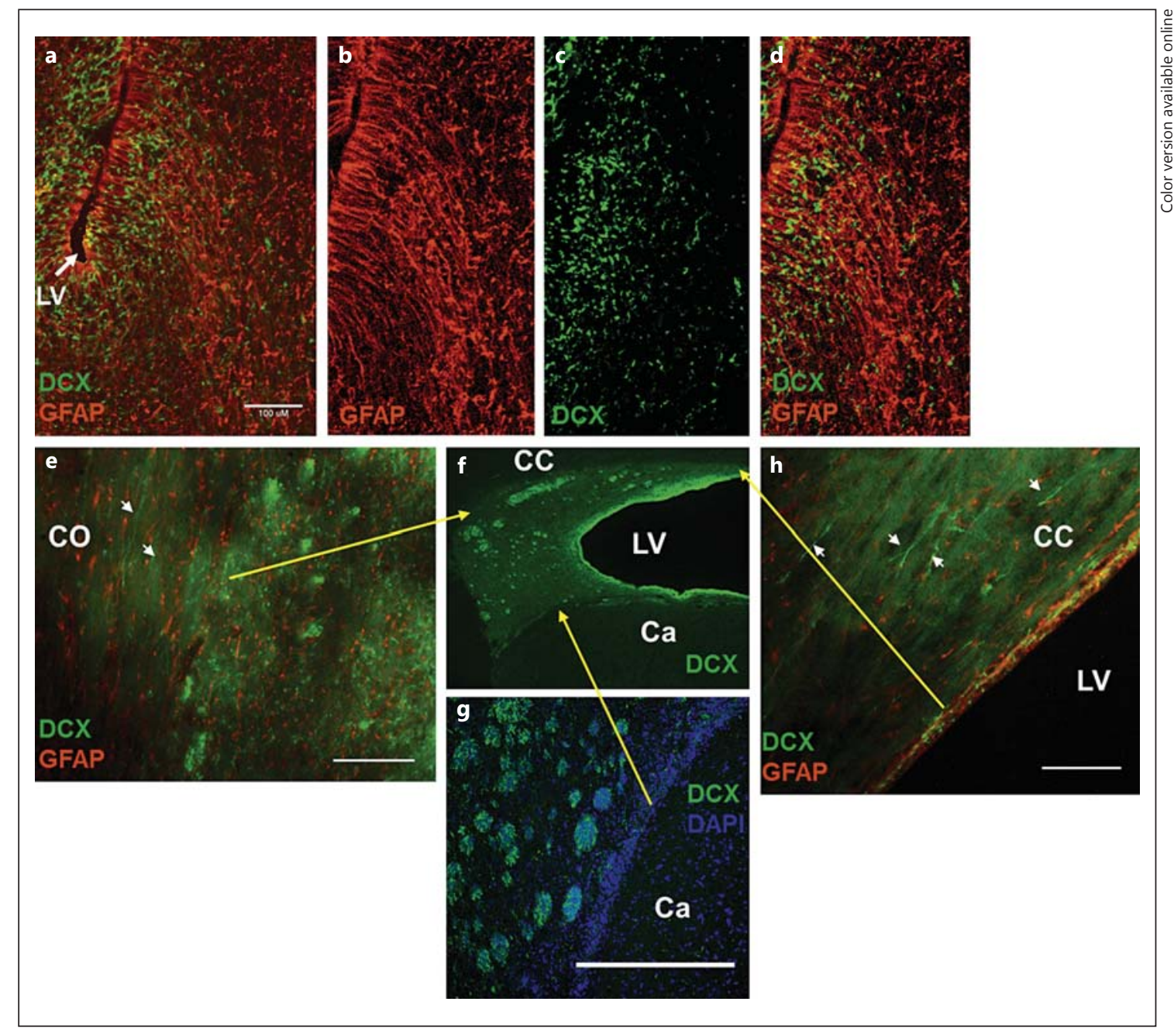

Fig. 3. Potential radial glia and variation in SVZ borders of the PND14 uninjured piglet. $\mathrm{CO}=$ Centrum semiovale (see fig. 1 for other abbreviations). Potential radial glia positive for GFAP are observed in a subset of piglets $(\mathbf{a}, \mathbf{b})$ radiating from the lateral ventricle out through the SVZ. c, d Some DCX+ neuroblasts appear to be associated with radial glia. The border of the SVZ with surrounding brain regions depends on location (location of borders indicated by yellow arrows to f). e The dorsal border of the SVZ with the centrum semiovale is somewhat indistinct and determined by the absence of

exist ventrally as chains of putative migrating neuroblasts branch off from the RMS ventrally in the internal capsule, though their destination is not clear (fig. $4 b, c, e$ ). In the extreme rostral RMS (L $4.0 \mathrm{~mm}$ ) neuroblasts appear to branch off from the RMS just dorsal to the olfactory ventricle and project rostrally into a gyrus of the frontal cortex in the gyral white matter tract (fig. $4 \mathrm{~b}, \mathrm{c}, \mathrm{h}$ ). In the caudal portion of the lateral ventricle adjacent to the dorsal-lateral hippocampus very large neuroblast chains are present in a potential migratory stream (fig. $4 \mathrm{~b}, \mathrm{j}$ ). In coronal sections neuroblast chains, less individually migrating neurons and less dense glia (GFAP+) in the centrum semiovale. The dorsolateral border was characterized by an abundance of individual DCX + cells with migratory morphologies in the centrum semiovale white matter adjacent to the SVZ (e, white arrows). $\mathbf{g}$ The border of the SVZ with the caudate is distinct. $\mathbf{h}$ Moving medially along the lateral ventricle, the Asvz expansion is no longer present and the Vsvz is thin. $\mathbf{h}$ Putative individually migrating neuroblasts can be seen in the adjacent corpus callosum (white arrows). Scale bars $=100 \mu \mathrm{M}$.

encompassing the lateral ventricle, the funnel point of the SVZ appears to be an additional exit point for neuroblasts populating lateral gray matter regions.

\section{Effects of Age on Anatomy and Trauma on the SVZ among Developmental Stages of Immaturity}

Piglets received cortical impact scaled to the size of the brain on the right rostral gyrus at PND 7, 1 month and 4 months of age, and the brain was collected 7 days after injury. The area of the Vsvz (fig. 5f, red) and neuroblast 


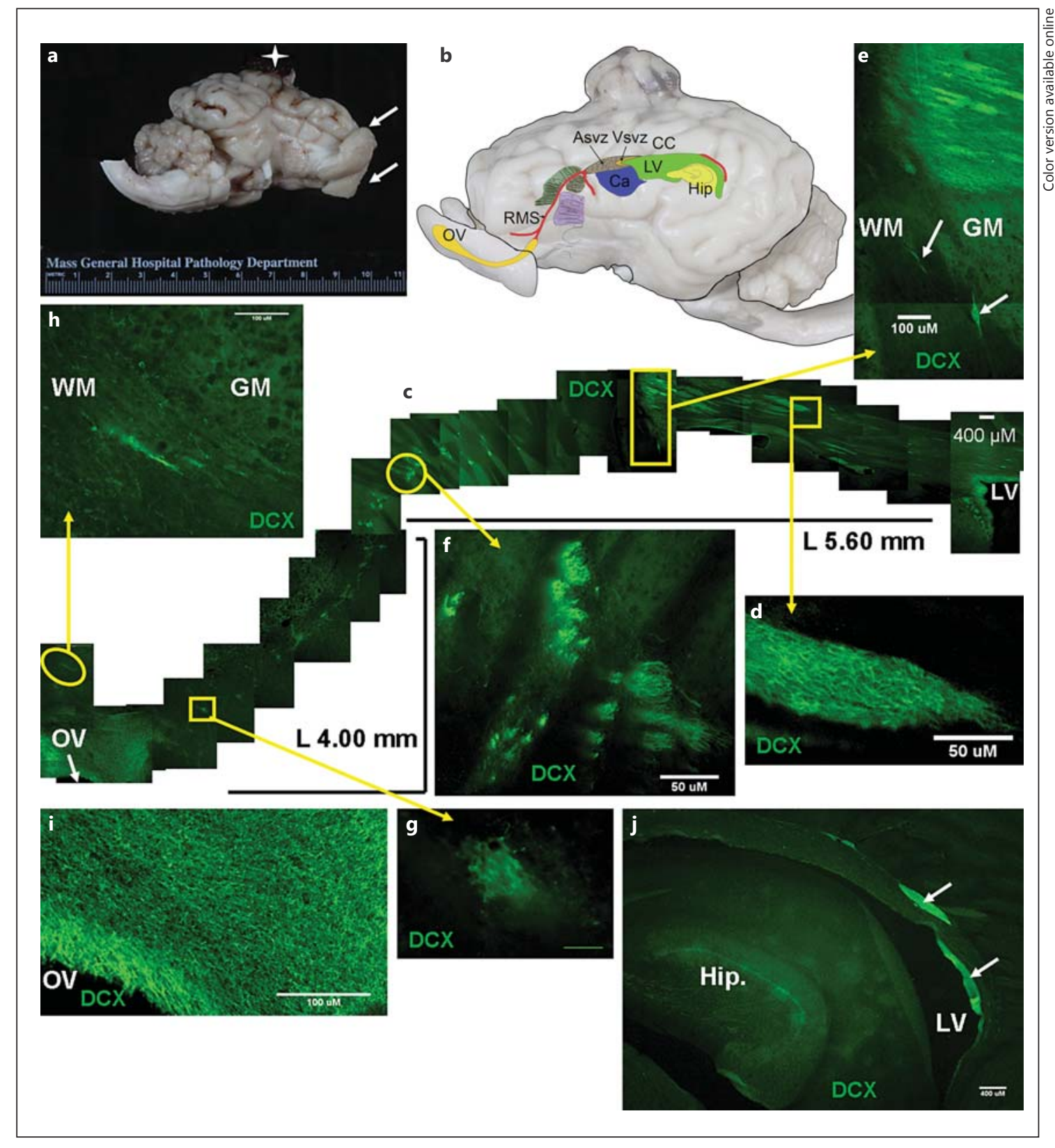

Fig. 4. The RMS and other potential streams in the uninjured PND14 piglet. $\mathrm{WM}=$ White matter; $\mathrm{GM}=$ gray matter; hip. = hippocampus; OV = olfactory ventricle; RMS = rostral migratory stream. (see fig. 1 for other abbreviations). a The piglet has large olfactory bulbs in proportion to the brain (injured 1-month-old piglet, white arrows, star = lesion). $\mathbf{b}$ Illustration of the course of the RMS from the SVZ to the olfactory ventricle. In the uninjured PND-14 piglet, from the RMS, a path branches off ventrally, a path branches off rostrally, and a path is present dorsal to the hippocampus (star = lesion). c From the lateral ventricle, large chains of neuroblasts appear to travel over the caudate and then narrow as they descend between the claustrum and internal capsule/putamen at L $5.60 \mathrm{~mm}$. After the putamen, the RMS descends laterally to $\mathrm{L} 4.00 \mathrm{~mm}$ to meet the ex- tension of the olfactory ventricle, which projects dorsally at this sagittal plane. d Higher magnification image of neuroblast chains appearing to move rostrally in the Asvz. e Higher magnification image of a potential ventral path in $\mathbf{c}$. $\mathbf{f}$ Higher magnification images of neuroblast chains descending around the putamen. $\mathbf{g}$ Higher magnification image of small neuroblast chains in the white matter tract leading to the olfactory ventricle. $\mathbf{h}$ Higher magnification image of small neuroblast chain appearing to migrate past the olfactory bulb in the white matter of a gyrus rostral to the olfactory ventricle. i Higher magnification image of the dense network of neuroblasts around the olfactory ventricle. $\mathbf{j}$ Large chains of neuroblasts (arrows) dorsal to the hippocampus adjacent to the lateral ventricle. Scale bars: $\mathbf{c}, \mathbf{j}=400 \mu \mathrm{M} ; \mathbf{e}, \mathbf{h}, \mathbf{i}=100 \mu \mathrm{M} ; \mathbf{d}, \mathbf{f}=50 \mu \mathrm{M} ; \mathbf{g}=10 \mu \mathrm{M}$. 


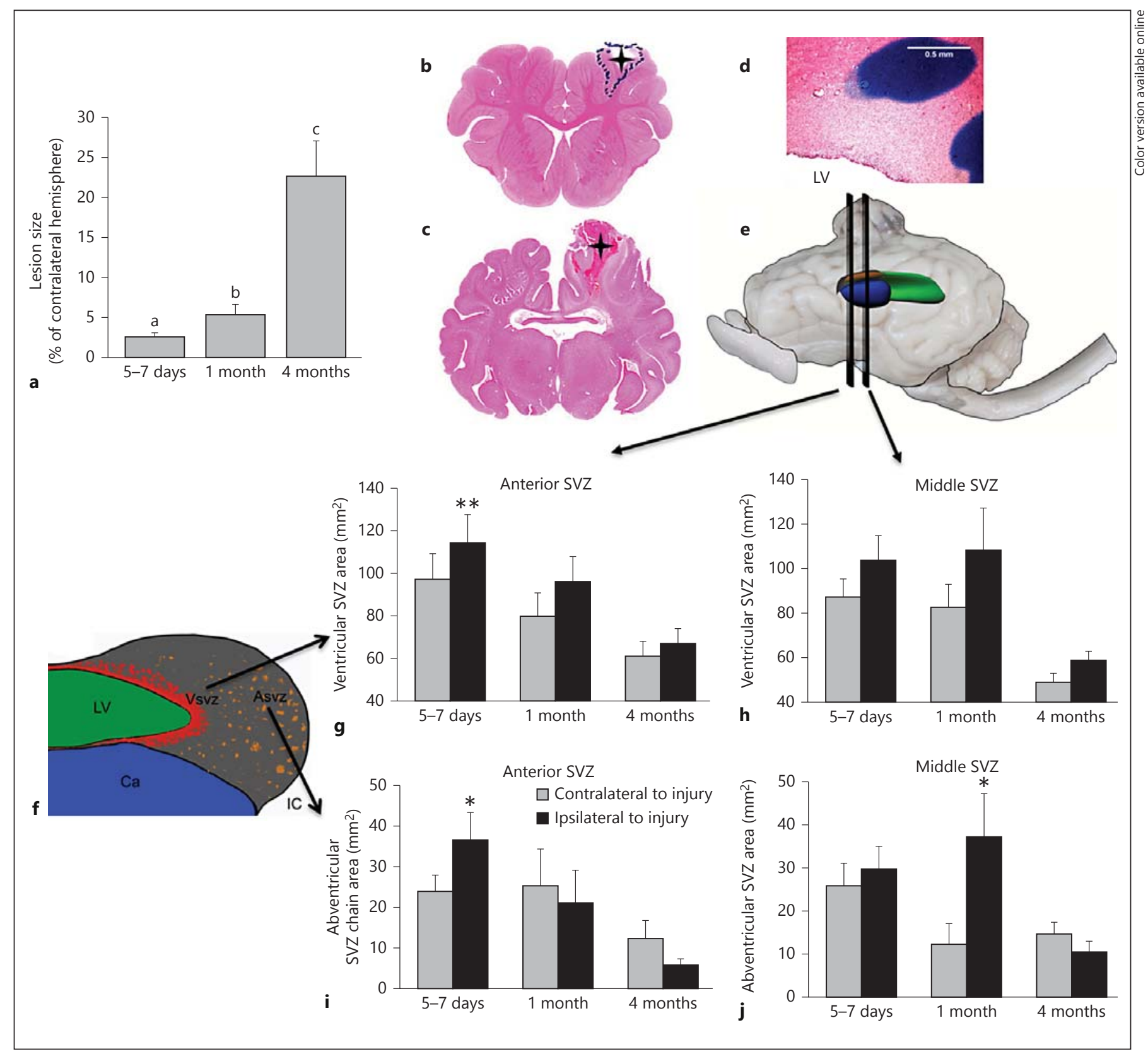

Fig. 5. Lesion size and dynamics of the SVZ after cortical impact in piglets among developmental stages of immaturity (see fig. 1 for abbreviations). The right rostral gyrus of the cortex was injured by impact scaled to the size of the brain in piglets at PND 5-7, 1 month or 4 months of age $(n=40)$. The brain was collected 7 days after injury. Lesion size was quantified on five $10-\mu \mathrm{M}$ coronal sections centered over the largest portion of the lesion. a In response to an injury scaled to the size of the brain, lesion size increased with age (differences tested via Student's t tests; means with different letters differ $\mathrm{p}<0.05$ $(\mathbf{a}-\mathbf{c})$ ). Lesions (outlined with dots, indicated by a star) were smallest in piglets injured on PND 5-7 (b) and were largest in piglets injured at 4 months of age (c), sometimes extending to the SVZ (d). In sections containing the anterior or middle SVZ (e), the area of the Vsvz (red in $\mathbf{f} ; \mathbf{g}, \mathbf{h}$ ) and Asvz (orange in $\mathbf{f} ; \mathbf{i}, \mathbf{j}$ ) neuroblast chains were quantified and the main effects of age, hemisphere and the interaction were tested via a two-way ANOVA followed by Student's t tests. The area of the Vsvz and the neuroblast chains in the Asvz both declined with age (main effect of age; $\mathbf{g - j}$ ). In piglets injured at PND $5-7$, the areas of both the anterior Vsvz and anterior Asvz neuroblast chains were greater in the hemisphere ipsilateral vs. contralateral to the injury $(\mathbf{g}, \mathbf{i})$. In piglets injured at 1 month of age, middle Asvz neuroblast chains were greater ipsilateral vs. contralateral to injury (j). No differences in the Asvz or Vsvz were observed in piglets injured at 4 months $(\mathbf{g}-\mathbf{j}) .{ }^{*} \mathrm{p}<0.05$ : means \pm SEM differ from the contralateral hemisphere within the same age group; ${ }^{* *} \mathrm{p}=0.07$ : means \pm SEM tended to differ from the contralateral hemisphere within the same age group. Scale bar $=0.5 \mathrm{~mm}$. 


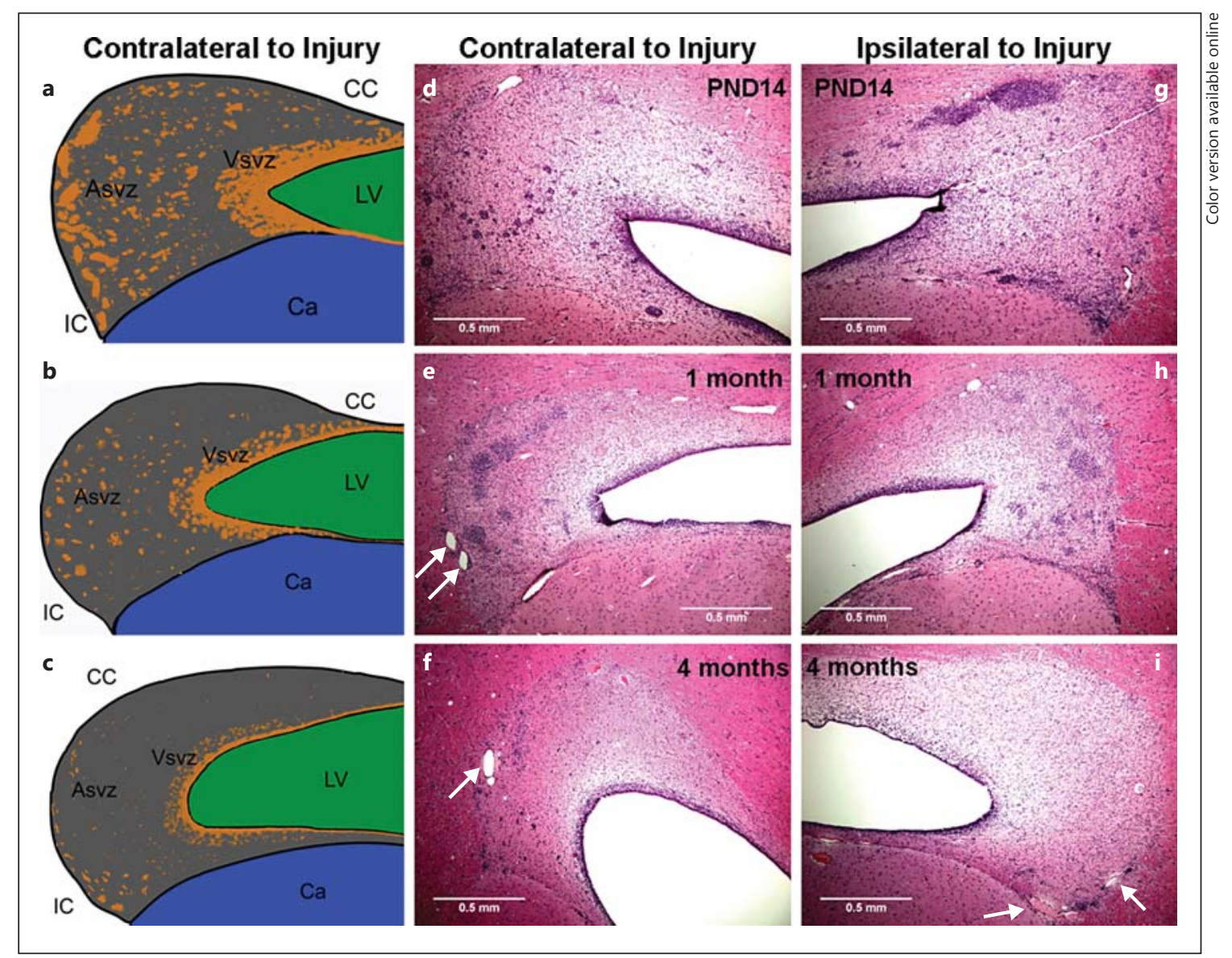

Fig.6. The effect of age and cortical impact on the anatomy of the piglet SVZ among developmental stages of immaturity (see fig. 1 for abbreviations). The right rostral gyrus of the cortex was injured by an impact scaled to the size of the brain in piglets at PND 5-7, 1 month or 4 months of age. The brain was collected 7 days after injury. The general anatomy of the SVZ did not differ among ages (a-f, contra- lateral to injury) or due to injury (g-i, ipsilateral to injury), but the area of Vsvz and Asvz neuroblast chains declined with age (hematoxylin, $\mathbf{d}-\mathbf{f}$ ). a-c Schematics illustrate differences in the SVZ among ages (green: lateral ventricle; blue: caudate; orange: Vsvz, Asvz). Blood vessels are abundant on the borders of the Asvz and qualitatively increase with age (white arrows, $\mathbf{d - f}$, i). Scale bar $=0.5 \mathrm{~mm}$. chains in the Asvz (fig. 5f, orange) were measured and compared among ages in the anterior or middle coronal plane (fig. 5e), containing both the SVZ and the lesion in the hemisphere ipsilateral or contralateral to the injury. Examining the hemisphere contralateral to the injury the architectural anatomy of the SVZ did not differ among ages (fig. 6a-f). Qualitatively, blood vessels (white arrows) appeared larger with increasing age (fig. $6 \mathrm{~d}-\mathrm{f}$, i). The areas of the Vsvz and Asvz declined with age (anterior Vsvz, $\mathrm{p}=0.0042$; fig. 5g; anterior Asvz, $\mathrm{p}=0.0004$; fig. 5i; middle Vsvz, $\mathrm{p}=0.0031$; fig. 5h; middle Asvz, $\mathrm{p}=$ 0.0160 ; fig. $5 j$ ). In piglets in which the SVZ was analyzed the lesion size expressed as a ratio to the uninjured hemisphere increased with increasing age (fig. $5 \mathrm{a}-\mathrm{d}$ ), with the largest lesions in the 4-month-old age group that some- times extended to the SVZ (fig. 5c, d). Piglets in which the lesion extended into the SVZ were excluded from analysis. In all ages the area of the Vsvz was greater than the total area of neuroblast chains in the Asvz ( $p<0.001$; fig. $5 g-j$ ).

In response to injury, the areas of the SVZ exhibited an age-dependent effect of trauma, with the younger ages having the greatest response to injury. In the anterior SVZ, the Vsvz area tended to be greater $(\mathrm{p}=0.07)$, and the area of the neuroblast chains in the Asvz were greater $(\mathrm{p}=0.045)$ in the hemisphere ipsilateral compared to contralateral to the injury in piglets injured at PND 7 (fig. 5g, i). In piglets injured at 1 month of age the area of the neuroblast chains of the middle Asvz was greater in the hemisphere ipsilateral versus contralateral to the in- 


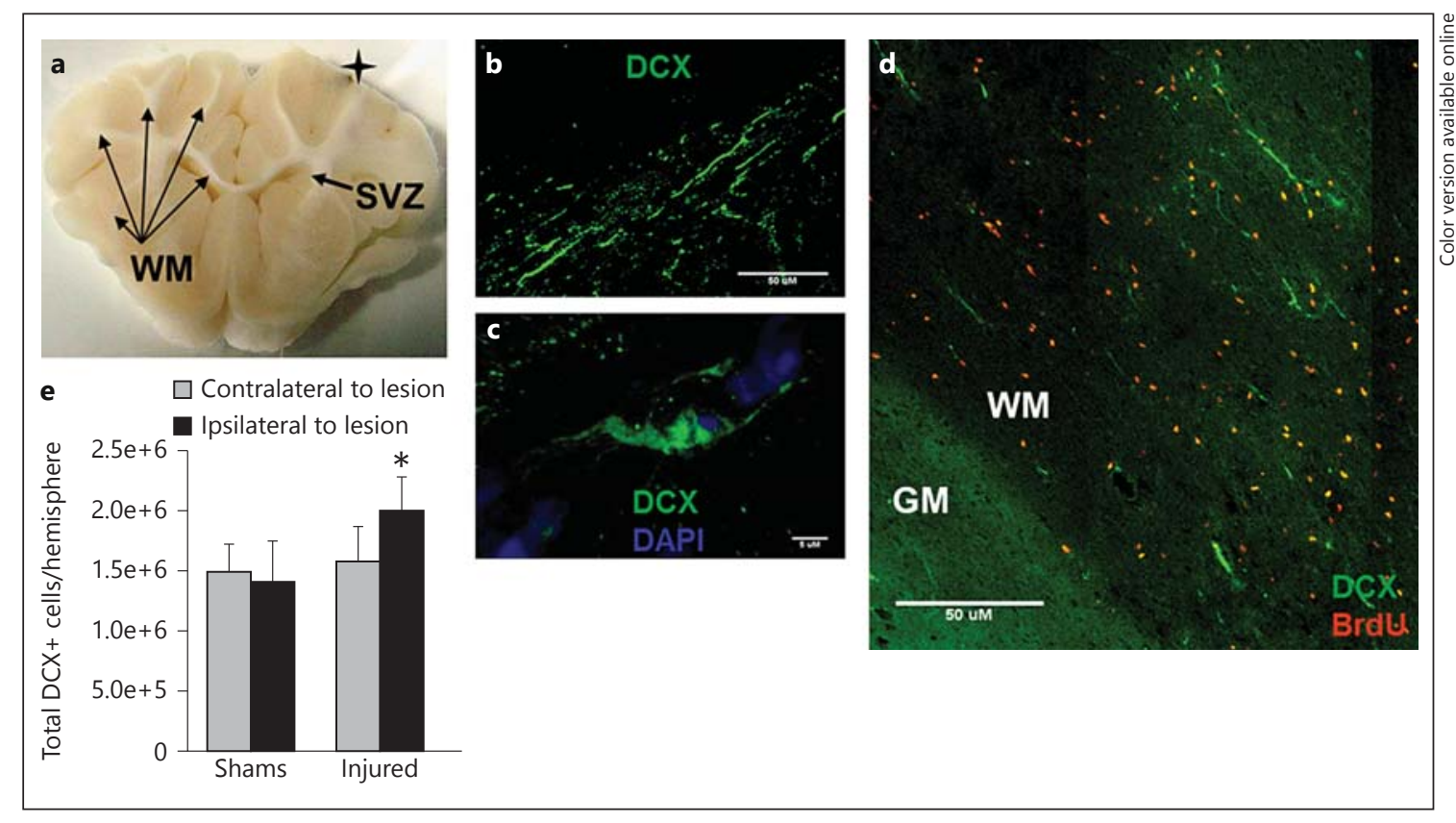

Fig. 7. Neuroblasts in the white matter tracts in PND-14 piglets that received cortical impact or sham surgery at PND 5-7. WM = White matter; GM = gray matter. The right rostral gyrus of the cortex was injured or a sham surgery was performed in piglets at PND 5-7 ( $\mathrm{n}=14)$. BrdU was injected after injury (PND 7-9) and brains were collected 1 week after injury. DCX + and DCX $+/ B r d U+$ colabeled neuroblasts were quantified via stereology in the white matter tracts (a) of the piglet in coronal $50-\mu \mathrm{M}$ sections underlying the lesion. As the piglet has extensive gyral white matter, neuroblasts generated in the SVZ would be required to travel through

jury $(p=0.0084$; age $\times$ side interaction, $p=0.0274$; fig. $5 j)$. At 4 months of age the area of neuroblast chains in the Asvz and the area of the Vsvz did not alter in response to injury (fig. $5 g-j$ ).

\section{Neuroblasts in the White Matter and the Effect of}

Trauma in PND-14 Piglets

In gyrencephalic species, if neuroblasts produced in the SVZ migrate to a lesion in the cortex they would be expected to migrate through the white matter of the corpus callosum, centrum semiovale, and gyral white matter tracts. The possibility that a cortical impact might increase the number of neuroblasts in the white matter was investigated in the youngest group. PND-7 piglets received a cortical impact to the rostral gyrus or sham surgery and BrdU on days 7-9 after injury. Brains were collected 7 days after injury (at PND 14) and neuroblasts were quantified in the extensive white matter tracts of the hemisphere ipsilateral or contralateral to injury or in the corresponding hemispheres in control animals (fig. 7a). gyral white matter to reach the lesion (a, star). An estimated $2.90 \pm$ 0.481 million putative migrating neuroblasts were observed in all white matter tracts $(\mathbf{b}, \mathbf{c})$ of the whole brain, but less than $1 \%$ of these colabeled with BrdU (d, gray matter). e Injured piglets did not have a greater number of neuroblasts in the white matter ipsilateral vs. contralateral or in comparison to sham piglets (unpaired Students t test); however, within the injured piglets, the number of neuroblasts in the white matter was greater in the hemisphere ipsilateral vs. contralateral to the injury (paired Student's t test). ${ }^{*} \mathrm{p}<$ 0.05: means \pm SEM differ. Scale bars: $\mathbf{b}, \mathbf{d}=50 \mu \mathrm{M} ; \mathbf{c}=5 \mu \mathrm{M}$.

Control piglets (PND 14) had a large population of putative individually migrating neuroblasts throughout the white matter in the coronal plane, with an estimated total of $2.90 \pm 0.481$ million cells. In contrast to neuroblasts that were restricted to discrete migratory streams in the sagittal plane, individually migrating neuroblasts were seen throughout the white matter in the coronal plane dorsal to the SVZ (fig. $7 \mathrm{~b}-\mathrm{d}$ ). Less than $1 \%$ of migrating neurons in the white matter were colabeled with BrdU injected after injury (fig. 7d).

In PND-7 piglets, there was a tendency for injured subjects to have a greater number of DCX+ neuroblasts migrating through the white matter of the ipsilateral compared to contralateral hemisphere than control subjects $(p=0.07$; fig. $7 e)$, though there was no evidence of a generalized increase due to trauma, as the total number (ipsilateral + contralateral hemisphere) of estimated migrating neurons was not different between control and injured piglets $(2.904 \pm 0.481$ vs. $3.577 \pm 0.565$ million, $\mathrm{p}=0.44)$. In injured piglets the number of DCX+ neurons 
in the white matter was greater in the hemisphere ipsilateral versus contralateral to the injury ( $\mathrm{p}=0.005$; fig. $7 \mathrm{e}$ ). There was no effect of injury or hemisphere in relation to injury on the percentage of DCX+ cells colabeled with BrdU (control ipsilateral: $0.21 \pm 0.06 \%$, control contralateral: $0.5 \pm 0.4 \%$, injured ipsilateral: $0.58 \pm 0.10 \%$, injured contralateral: $0.82 \pm 0.24 \%)$. Therefore, the vast abundance of putative migrating neuroblasts in the white matter of PND-14 piglets was generated constitutively regardless of injury.

\section{Discussion}

Here we describe the anatomy of the SVZ in the piglet for the first time. In addition to the SVZ proper (Vsvz), piglets have an extensive Asvz laterally, with an abundance of neuroblast chains similar to human infants and rabbits, in which this expansive portion is considered part of the SVZ $[4,5]$. As has been established in adult pigs, the enlarged Asvz is continuous with the Vsvz [8]. Piglets at PND 14, 1 month and 4 months of age displayed similar anatomy, though the area of the neuroblast chains in the Vsvz and Asvz declined by $50 \%$ between the age of 2 weeks and 4 months (prepubertal). This apparent reduction of tissue volume associated with active neurogenesis as piglets age is similar to studies in Göttingen minipigs, where the number of proliferating cells, as detected by BrdU, in the SVZ and dentate gyrus declines by $50-60 \%$ between $6-7$ weeks and 8 months of age (postpubertal), though these single-labeled BrdU+ cells can give rise to glia, oligodendrocytes or neurons [7].

In the piglet the neuroblast chains in the Vsvz lie directly under the ependymal cells, as observed in human infants up to 6 months of age [5], immature dogs [31], rabbits [4], and mice [32]. A distinct hypocellular layer containing only glial cell processes and devoid of either GFAP+ or DCX + cell bodies was not observed at any age of piglets examined, as is observed in the SVZ of human infants over 6 months of age coincident with the migration of this population to the prefrontal cortex [5]. Radial glia or radial-like glia oriented from the ventricle and projecting out through the SVZ were observed in some subjects on PND 14, as detected by GFAP. Similarly, radial glia were only observed early in postnatal development in rabbits [4] and humans [5] but persisted past immaturity in the dog and mouse $[33,34]$. The pattern and orientation of these cells in the piglet are similar to radial-like glia described in the SVZ surrounding the ventral lateral ventricle in adult mice [34]. A proportion of DCX+ cells appear to orient with the radial glia. Radial glia, as detected by GFAP, were only observed in some piglets at PND 14, perhaps indicating the initiation of regression of radial glia or the initiation of expression of GFAP by radial glia.

The DCX+ chains in the Asvz observed in the piglet are similar to those observed in the immature human SVZ [5]. In piglets these chains are prominent in all ages examined, including the preadolescent, whereas they are not observed after 18 months of age in human toddlers $[5,8]$. The prolonged persistence of chains in piglets beyond the equivalent developmental stage in humans may be due to piglets being macrosmatic animals. Neuroblast chains located in the Asvz are not observed in rodents, dogs or cats at any age $[3,32]$. In lagomorphs, in which the olfactory ventricle remains continuous with the lateral ventricle postnatally, chains of neuroblasts are seen both in the Vsvz and Asvz from birth through PND 30 [4]. In the current study, sagittal sections reveal that the chains of neuroblasts viewed in the coronal plane in the dorsal SVZ appear to be migrating chains of neuroblasts oriented anterior-posterior towards the RMS. However, neuroblast chains in the ventral Asvz do not appear to be oriented in a specific direction but appear round in both sagittal and coronal planes. The movement pattern, relative proliferation and final destination of neuroblast chains in the ventral Asvz are unknown. The contact of neuroblast chains to glial cells observed in the Asvz in piglets are in a similar arrangement to human infants and immature rabbits, having loose contact with astrocytes, thus allowing the potential for exposure to signals delivered through blood vessels or from the white matter [4, 5]. Glial tubes observed in rodents were not observed in the piglet [35]. Glial contact with neuroblast chains in the Asvz is more extensive in the ventral than in the dorsal Asvz. The open structure of these chains is purported to allow for plasticity and responsiveness to cues for migration other than automated migration to the RMS and allow migration relatively independent of astrocytes [4].

The RMS of the piglet followed a similar path, as described in postnatal cats, dogs and rodents $[3,36]$. In contrast to human infants where both the lateral ventricle and the RMS deflect ventrally immediately caudal to the caudate, the anterior tip of the lateral ventricle and proximal RMS of the piglet do not deflect ventrally but move rostrally before the RMS deflects ventrally rostral to the caudate. In the piglet, both chains of neuroblasts and neuroblasts in an individual migratory phenotype travel along the RMS, moving laterally before moving into the olfactory bulb. Potential additional pathways of putative migrating chains were observed - diverging from the RMS and projecting ventrally through the internal cap- 
sule, posterior along the lateral ventricle dorsal to the hippocampus and branching off the RMS into the white matter tract rostral to the olfactory ventricle. The ventral stream might be comparable to the ventral migratory stream described in rodents, in which the ventral migratory mass migrates from the SVZ to the nucleus accumbens where it forms the islands of Calleja [36, 37]. Certainly, in the uninjured PND-14 piglet brain, similar to the human infant brain, multiple brain regions are actively being populated with neuroblasts from the SVZ and the migration of neuroblasts does not appear to be restricted to the olfactory bulb.

In response to injury, the SVZ underwent dynamic changes that were age dependent. In this previously characterized model of TBI scaled among stages of immaturity, the youngest age group (infants, PND 7) had the smallest lesions, while the oldest piglets (preadolescents, 4 months of age) had large lesions that may expand into the burr hole of the skull and extend into the white matter and, in severe cases, into the SVZ [23]. Piglets at a stage of development similar to human toddlers (1 month of age) have an intermediate response [23]. We hypothesized that developmental differences in the SVZ and a neurogenic response contributed to the age-dependent response to injury and, ultimately, lesion size. The effect of injury on the SVZ was most pronounced in piglets injured at PND 7, exhibiting an increase of both the Vsvz and Asvz neuroblast chains 1 week after injury in the ipsilateral compared to contralateral hemisphere. Piglets injured at 1 month of age had an increase in the area of Asvz neuroblast chains only, while piglets injured at 4 months had no response to injury. One limitation of this study is the comparison of the SVZ between the ipsilateral and contralateral hemisphere among piglets that were all injured. If the injury affected the contralateral hemisphere in addition to the ipsilateral hemisphere, our observed effect of TBI on the SVZ might be more blunted than if the injured piglets were compared to sham piglets. Regardless of the observed changes in the SVZ in PND-14 piglets, TBI did not result in a greater number of neuroblasts in the white matter born before or after injury compared to sham piglets. While neurogenesis and the SVZ have been well studied in adult rodent models of TBI [16, $26,38-40]$, the effect of TBI on the SVZ and neuroblasts in the white matter among stages of immaturity has received limited attention [17]. The response of the SVZ to TBI in piglets is limited compared to adult rodents and might reflect differences in the response of injury in largebrained species or a developmental effect where the immature brain utilizes existing neuroblasts for lesion re- pair. To date, studies focusing on the effect of age on neurogenesis after injury primarily examine the effect of hypoxic-ischemic injury and/or examine the dentate gyrus of the hippocampus, and these results are difficult to translate among different injury types and/or brain regions $[17,41,42]$. The effects of TBI on the SVZ and the migration of neuroblasts through the white matter superimposed upon the developmental ontogeny of neurogenesis certainly merit further study.

Surprisingly, the number of DCX + cells or DCX + / BrdU colabeled cells in the white matter of PND-14 piglets was not increased in the injured compared to sham piglets. This is in contrast to rodents in which the injured striatum contains an abundance of neuroblasts as early as 5 days after injury [13]. However, it has been demonstrated that the neuroblasts that migrate to the injury were born in the 2 days prior to injury [13]. The time required for neuroblasts to migrate to the injured cortex in this large animal model requires further study, as the generation of new neuroblasts and migration to the site of injury in large-brained species may require more time than in rodent species. This would not be surprising, as neuroblasts generated in the SVZ would need to travel through the corpus callosum, centrum semiovale and gyral white matter to reach a lesion in the cortex in both human children and piglets. To our knowledge, this is the first attempt to quantify neuroblasts in the centrum semiovale and gyral white matter after a cortical injury.

Alteration in migration patterns of neuroblasts after TBI may impact neural developmental ontogeny. Indeed, 'being plastic' may not always be 'fantastic' [43], but alteration of neuroblast migration may cause longterm deficits and contribute to the phenomena of growing into deficits after head injury in children. Three additional neural transit pathways from the SVZ during discrete periods of postnatal neurodevelopment have been described in other species, as follows: (1) the medial migratory stream that populates the prefrontal cortex in infants [5], (2) the ventral migratory mass that migrates along the ventrocaudal migratory stream and populates the Islands of Calleja [36, 37] and (3) the dorsal migratory pathway dorsal to the hippocampus directed towards the occipital cortex [36]. PND-14 piglets had a large amount of putative migrating neuroblasts throughout the white matter in the coronal plane dorsal to the SVZ, while neuroblasts in the sagittal plane were restricted to discrete migratory pathways. However, TBI did not increase the overall abundance of neuroblasts in white matter, but neuroblasts might have been directed to the injured hemisphere. In human infants and children, in 
an acute effort to repair lesioned tissue after TBI, migrating neuroblasts may be redirected at the expense of other brain regions programmed to populate in postnatal life. If the absolute number of neuroblasts is increased only to a limited degree in this age group, the redirection of neuroblasts from the SVZ intended for the prefrontal cortex might impact the development of higher executive function. Neuroblasts intended for the nucleus accumbens might be redirected, potentially inducing lifelong effects on reward circuitry, anxiety and aggression. Indeed, aberrant postnatal migration of neurons to these regions has been hypothesized to contribute to cognitive deficits associated with schizophrenia [44]. In humans, it is not yet clear whether migration is directed to the injured brain region [17] and/or whether abnormally migrating neuroblasts develop into a substrate for seizures. Aberrant migration as observed in cortical dysplasias is associated with seizures [45], and ectopic neurons have been observed in children after TBI [17]; however, cause and effect has yet to be established. In our large animal model, it is not yet known whether these detoured neuroblasts arrive at the lesioned area and, if so, whether they survive and integrate.

In conclusion, the anatomy of the SVZ of piglets shares many characteristics with the SVZ in human infants, and the inherent repair strategy in response to TBI appears to be age specific. Piglets injured at PND 7, which have the smallest lesions [23], have the largest SVZ, and the SVZ areas increased in response to injury. At this age, addi- tional neuroblasts might be targeted to the lesion without requiring upregulation of neurogenesis. In piglets injured at 4 months of age, which have the largest lesions [23], SVZ areas are smaller at baseline and did not increase in response to TBI. It remains to be determined whether neuroblasts specifically target injured tissue in the PND-7 piglets and whether there is an increase in the number of migrating neuroblasts in 1- and 4-month-old pigs. Exploration into the strategies employed by the porcine brain for self-repair and the long-term consequences of acute repair responses might reveal new therapeutic age-specific targets for repair after TBI in infants and children that enhance the restoration of the injured tissues while preserving normal neural development.

\section{Acknowledgments}

This work was supported by R01 HD45364-5 from the NIH National Institute of Child Health and Human Development, the Pediatric Neuroscience Fund of the Children's Hospital at Dartmouth, the Nicholas T. Zervas Chair of Harvard Medical School, and the Joel Alan Viktor Research Fund at Massachusetts General Hospital. The authors express their gratitude to Leslie Adams, Kate Hillier, Ijeoma Chinwuba, Michael Simoni, and Kristen Saliga for their assistance with animal handling, immunohistochemistry and quantification. The authors also wish to thank Kenneth Orndorff (Dartmouth Optical Cellular Imaging Shared Resource Center) and Igor Bagayev (MGH Neuroscience Center Imaging Core supported by NINDS grant 5P30NS045776) for their assistance with confocal microscopy and Emily Belli for proofreading the manuscript.

\section{References}

1 Breunig JJ, Arellano JI, Macklis JD, Rakic P: Everything that glitters isn't gold: a critical review of postnatal neural precursor analyses. Cell Stem Cell 2007;1:612-627.

- Altman J: Autoradiographic and histological studies of postnatal neurogenesis. IV. Cell proliferation and migration in the anterior forebrain, with special reference to persisting neurogenesis in the olfactory bulb. J Comp Neurol 1969;137:433-457.

- 3 Malik SZ, Lewis M, Isaacs A, Haskins M, Van Winkle T, Vite $\mathrm{CH}$, et al: Identification of the rostral migratory stream in the canine and feline brain. PLoS One 2012; 7:e36016.

4 Ponti G, Aimar P, Bonfanti L: Cellular composition and cytoarchitecture of the rabbit subventricular zone and its extensions in the forebrain. J Comp Neurol 2006;498:491-507.

-5 Sanai N, Nguyen T, Ihrie RA, Mirzadeh Z, Tsai HH, Wong M, et al: Corridors of migrating neurons in the human brain and their decline during infancy. Nature 2011;478:382386.
6 Yin F, Guo L, Lu RF, Zhu QS: Spontaneous differentiation of porcine neural progenitors in vitro. Cytotechnology 2011;63:363-370.

7 Guidi S, Bianchi P, Alstrup AK, Henningsen K, Smith DF, Bartesaghi R: Postnatal neurogenesis in the hippocampal dentate gyrus and subventricular zone of the Göttingen minipig. Brain Res Bull 2011;85:169-179.

8 Liard O, Segura S, Pascual A, Gaudreau P, Fusai $\mathrm{T}$, Moyse E: In vitro isolation of neural precursor cells from the adult pig subventricular zone. J Neurosci Methods 2009; 182:172179.

-9 Longhi L, Watson DJ, Saatman KE, Thompson HJ, Zhang C, Fujimoto S, et al: Ex vivo gene therapy using targeted engraftment of NGF-expressing human NT2N neurons attenuates cognitive deficits following traumatic brain injury in mice. J Neurotrauma 2004; 21:1723-1736.

10 Masuda T, Isobe Y, Aihara N, Furuyama F, Misumi S, Kim TS, et al: Increase in neurogenesis and neuroblast migration after a small intracerebral hemorrhage in rats. Neurosci Lett 2007;425:114-119.

-11 Jin K, Wang X, Xie L, Mao XO, Greenberg DA: Transgenic ablation of doublecortinexpressing cells suppresses adult neurogenesis and worsens stroke outcome in mice. Proc Natl Acad Sci U S A 2010;107:79937998.

12 Nixon K, Kim DH, Potts EN, He J, Crews FT: Distinct cell proliferation events during abstinence after alcohol dependence: microglia proliferation precedes neurogenesis. Neurobiol Dis 2008;31:218-229.

13 Gordon RJ, Tattersfield AS, Vazey EM, Kells AP, McGregor AL, Hughes SM, et al: Temporal profile of subventricular zone progenitor cell migration following quinolinic acid-induced striatal cell loss. Neuroscience 2007; 146:1704-1718.

14 Covey MV, Jiang Y, Alli VV, Yang Z, Levison SW: Defining the critical period for neocortical neurogenesis after pediatric brain injury. Dev Neurosci 2010;32:488-498. 
15 Zhang C, Saatman KE, Royo NC, Soltesz KM, Millard M, Schouten JW, et al: Delayed transplantation of human neurons following brain injury in rats: a long-term graft survival and behavior study. J Neurotrauma 2005;22:14561474.

16 Saha B, Peron S, Murray K, Jaber M, Gaillard A: Cortical lesion stimulates adult subventricular zone neural progenitor cell proliferation and migration to the site of injury. Stem Cell Res 2013;11:965-977.

-17 Taylor SR, Smith C, Harris BT, Costine B, Duhaime AC: Maturation-dependent response of neurogenesis after traumatic brain injury in children. J Neurosurg Pediatr 2013;12:545554 .

18 Duhaime AC: Large animal models of traumatic injury to the immature brain. Dev Neurosci 2006;28:230-237.

$\rightarrow 19$ Statler KD: Pediatric posttraumatic seizures: epidemiology, putative mechanisms of epileptogenesis and promising investigational progress. Dev Neurosci 2006;28:354-363.

20 Margulies S, Hicks R: Combination therapies for traumatic brain injury: prospective considerations. J Neurotrauma 2009;26:925-939.

21 Dobbing J, Sands J: Comparative aspects of the brain growth spurt. Early Hum Dev 1979; 311:79-83.

22 Dickerson JWT, Dobbing J: Prenatal and postnatal growth and development of the central nervous system of the pig. Proc R Soc Lond B Biol Sci 1967;166:384-395.

23 Missios S, Harris BT, Dodge CP, Simoni MK, Costine BA, Lee YL, et al: Scaled cortical impact in immature swine: effect of age and gender on lesion volume. J Neurotrauma 2009;26: 1943-1951.

-24 Duhaime AC, Margulies SS, Durham SR, O'Rourke MM, Golden JA, Marwaha S, et al: Maturation-dependent response of the piglet brain to scaled cortical impact. J Neurosurg 2000;93:455-462.

25 Doetsch F, Garcia-Verdugo JM, AlvarezBuylla A: Cellular composition and three-dimensional organization of the subventricular germinal zone in the adult mammalian brain. J Neurosci 1997;17:5046-5061.
Gleason D, Fallon JH, Guerra M, Liu JC, Bryant PJ: Ependymal stem cells divide asymmetrically and transfer progeny into the subventricular zone when activated by injury. Neuroscience 2008;156:81-88.

27 Grate LL, Golden JA, Hoopes PJ, Hunter JV, Duhaime AC: Traumatic brain injury in piglets of different ages: techniques for lesion analysis using histology and magnetic resonance imaging. J Neurosci Methods 2003;123:201-206.

28 Koob AO, Harris BT, Duhaime AC: Cellular genesis in the postnatal piglet. Int J Dev Neurosci 2008;26:641-646.

29 Francis HW, Rivas A, Lehar M, Saito Y, Mouton PR, Ryugo DK: Efficient quantification of afferent cochlear ultrastructure using designbased stereology. J Neurosci Methods 2006; 150:150-158.

30 Felix B, Leger ME, Albe-Fessard D, Marcilloux JC, Rampin O, Laplace JP: Stereotaxic atlas of the pig brain. Brain Res Bull 1999;49: $1-137$.

-31 Blakemore WF, Jolly RD: The subependymal plate and associated ependyma in the dog. An ultrastructural study. J Neurocytol 1972;1:6984.

32 Jankovski A, Sotelo C: Subventricular zoneolfactory bulb migratory pathway in the adult mouse: cellular composition and specificity as determined by heterochronic and heterotopic transplantation. J Comp Neurol 1996;371: 376-396.

33 Walton RM, Parmentier T, Wolfe JH: Postnatal neural precursor cell regions in the rostral subventricular zone, hippocampal subgranular zone and cerebellum of the dog (Canis lupusfamiliaris). Histochem Cell Biol 2013;139: 415-429.

34 Sundholm-Peters NL, Yang HK, Goings GE, Walker AS, Szele FG: Radial glia-like cells at the base of the lateral ventricles in adult mice. J Neurocytol 2004;33:153-164.

35 Peretto P, Giachino C, Aimar P, Fasolo A, Bonfanti L: Chain formation and glial tube assembly in the shift from neonatal to adult subventricular zone of the rodent forebrain. J Comp Neurol 2005;487:407-427.

36 Inta D, Alfonso J, von Engelhardt J, Kreuzberg MM, Meyer AH, van Hooft JA, et al: Neurogenesis and widespread forebrain migration of distinct GABAergic neurons from the postnatal subventricular zone. Proc Natl Acad Sci U S A 2008;105:20994-20999.
37 De Marchis S, Fasolo A, Puche AC: Subventricular zone-derived neuronal progenitors migrate into the subcortical forebrain of postnatal mice. J Comp Neurol 2004;476:290300 .

38 Urrea C, Castellanos DA, Sagen J, Tsoulfas P, Bramlett HM, Dietrich WD: Widespread cellular proliferation and focal neurogenesis after traumatic brain injury in the rat. Restor Neurol Neurosci 2007;25:65-76.

39 Chirumamilla S, Sun D, Bullock MR, Colello $\mathrm{RJ}$ : Traumatic brain injury induced cell proliferation in the adult mammalian central nervous system. J Neurotrauma 2002;19:693703.

40 Thomsen GM, Le Belle JE, Harnisch JA, Mc Donald WS, Hovda DA, Sofroniew MV, et al: Traumatic brain injury reveals novel cell lineage relationships within the subventricular zone. Stem Cell Res 2014;13:48-60.

$\checkmark 41$ Qiu L, Zhu C, Wang X, Xu F, Eriksson PS, Nilsson $M$, et al: Less neurogenesis and inflammation in the immature than in the juvenile brain after cerebral hypoxia-ischemia. J Cereb Blood Flow Metab 2007;27: 785-794.

42 Sun D, Colello RJ, Daugherty WP, Kwon TH, McGinn MJ, Harvey HB, et al: Cell proliferation and neuronal differentiation in the dentate gyrus in juvenile and adult rats following traumatic brain injury. J Neurotrauma 2005; 22:95-105

43 Giza CC, Prins ML: Is being plastic fantastic? Mechanisms of altered plasticity after developmental traumatic brain injury. Dev Neurosci 2006;28:364-379.

-44 Gulsuner S, Walsh T, Watts AC, Lee MK, Thornton AM, Casadei S, et al: Spatial and temporal mapping of de novo mutations in schizophrenia to a fetal prefrontal cortical network. Cell 2013;154:518-529.

45 Boer K, Lucassen PJ, Spliet WG, Vreugdenhil E, van Rijen PC, Troost D, et al: Doublecortinlike (DCL) expression in focal cortical dysplasia and cortical tubers. Epilepsia 2009;50: 2629-2637. 